\title{
A THIRD ORDER BOUNDARY VALUE PROBLEM ARISING IN AEROELASTIC WING THEORY*
}

\author{
By GEORGE SEIFERT** (Cornell University)
}

The differential equation arising in the problems of chordwise divergence or sweptforward wing bending divergence [1] is of the type

$$
\frac{d^{2}}{d x^{2}} E I(x) \frac{d z}{d x}-\lambda c(x) z=0
$$

where $\lambda$ is a parameter. The boundary conditions are $z(0)=z^{\prime}(l)=\left(E I(x) z^{\prime}(x)\right)_{x=l}^{\prime}=0$. Since this represents a non self-adjoint boundary value problem, the ordinary RayleighRitz variational method for approximating its characteristic values is not applicable. Formal extensions of this variational approach have been suggested by Flax [1] and used by Cheng [2] but no mathematical evidence for their validity is at present available. In this connection, two questions have been raised [1].

(A) Under what conditions on $E I(x)$ and $c(x)$ are all the characteristic values of (1a) real?

(B) Under what conditions on $E I(x), c(x)$ and $f(x)$ can the solution $z_{\lambda}(x)$ of

$$
\left[E I(x) z^{\prime}(x)\right]^{\prime \prime}-\lambda c(x) z(x)=f(x), \quad z(0)=z^{\prime}(l)=\left(E I z^{\prime}\right)_{l}^{\prime}=0
$$

be expanded in terms of the biorthogonal system of functions arising from the system (1a) and its adjoint?

Although this paper concerns itself chiefly with (A), it may be pointed out that the investigations of expansions in terms of characteristic functions of (1a) for $E I \equiv 1$, $c(x) \equiv-1, l=\pi$ carried out by L. E. Ward [3] indicate difficulties inherent in such irregular boundary-value expansion problems as (B).

Part I of this paper deals with the characteristic values of the system

$$
u^{\prime \prime \prime}(x)+p(x) u^{\prime}(x)+[q(x)+\lambda] u(x)=0, \quad u(0)=u^{\prime}(0)=u^{\prime \prime}(1)=0,
$$

where $p$ and $q$ are real-valued functions analytic on $0 \leq x \leq 1$. It is found that:

(a) this system has an infinite number of real characteristic values (Theorem 1);

(b) if the upper bounds of $|p(t)|$ and $|q(t)|$, and the positive numbers $|p(1)|$, $\int_{0}^{1}|p(t)| d t, \int_{0}^{1}|r(t)| d t$, where $r(t)=q(t)-p^{\prime}(t)$, are small enough, then all characteristic values of (1) are real (Theorem 3 ).

Just how small these positive quantities in (b) must be, in order that all the characteristic values of (1) be real, is not explicitly considered. For specific functions $p$ and $q$, however, the details of the proofs of (a) and (b) enable one to answer this question by means of simple numerical computations.

Much of the method of proof and notation is similar to that used by Ward [4] in his study of the system:

$$
u^{\prime \prime \prime}(x)+\left[\rho^{3}+r(x)\right] u(x)=0, \quad u(0)=u^{\prime}(0)=u(\pi)=0 .
$$

Here $r(x)$ is of a special form which permits Ward to obtain an expansion theorem. It

*Received Oct. 17, 1950.

${ }^{* *}$ Now at the University of Nebraska. 
is hoped that Ward's approach will also suggest an expansion theorem associated with (1) and perhaps also provide an answer to (B).

Part II indicates a pair of transformations which takes the system

$$
\left[f(t) y^{\prime \prime}(t)\right]^{\prime}+\lambda g(t) y(t)=0, \quad y(0)=y^{\prime}(0)=y^{\prime \prime}(1)=0,
$$

where $f(t)>0, g(t)>0$, are real and analytic on $0 \leq t \leq 1$, over into a system of the form of (1). Note that the adjoint of (2) is of the form of (1a), and consequently the characteristic values of (2), being identical with those of its adjoint, are of interest in aeroelastic wing theory.

\section{PART I}

We consider the system (1). Define

(a) $\delta_{1}(t)=e^{\omega_{1} t}+e^{\omega_{2} t}+e^{\omega_{3} t}$

$$
\begin{aligned}
& \delta_{2}(t)=e^{\omega_{1} t}-\omega_{3} e^{\omega_{2} t}-\omega_{2} e^{\omega_{3} t} \\
& \delta_{3}(t)=e^{\omega_{1} t}-\omega_{2} e^{\omega_{2} t}-\omega_{3} e^{\omega_{0} t}
\end{aligned}
$$

where

$$
\omega_{1}=-1, \quad \omega_{2}=e^{(\pi i) / 3}, \quad \omega_{3}=e^{-(\pi i) / 3} ;
$$

(b) the complex number $\rho$ by $\rho^{3}=\lambda,|\arg \rho| \leq \pi / 3$;

(c) the regions $S_{1}$ and $S_{2}$ of the $\rho$ plane by $0 \leq \arg \rho \leq \pi / 3$ and $-\pi / 3 \leq \arg \rho \leq 0$ respectively.

Lemma 1. A necessary and sufficient condition that $u(x, \rho)$ satisfy the equation in (1) and $u(0, \rho)=u^{\prime}(0, \rho)=0^{1}$ is that

$$
u(x, \rho)=\delta_{3}(\rho x)-\left(3 \rho^{2}\right)^{-1} \int_{0}^{x}\left\{\delta_{3}[\rho(x-t)] r(t)-\rho \delta_{2}[\rho(x-t)] p(t)\right\} u(t, \rho) d t
$$

where $r(t)=q(t)-p^{\prime}(t)$.

The proof of this lemma is completely analogous to that of Theorem 1 of Ward's paper $^{2}$ and is omitted here.

Lemma 2. A solution $u(x, \rho)$ of the equation in (1) such that $u(0, \rho)=u^{\prime}(0, \rho)=0$ is given by

$$
u(x, \rho)=e^{\omega_{3} \rho x}\left[-\omega_{3}-\omega_{2} e^{\left(\omega_{2}-\omega_{s}\right) \rho x}+z(x, \rho)\right]
$$

where $|z(x, \rho)| \leq m, m$ being independent of $x$ and $\rho$, provided $\rho \epsilon S_{1}$ and $|\rho|$ sufficiently large.

${ }^{1}$ Unless otherwise indicated, the prime will always denote differentiation with respect to the first variable.

${ }^{2}$ L. E. Ward, [4], p. 418. 
Proof. Substituting (1.2) into (1.1) one obtains

$e^{\omega>\rho x}[A(x, \rho)+z(x, \rho)]$

$$
=\delta_{3}(\rho x)-\left(3 \rho^{2}\right)^{-1} \int_{0}^{x}\left\{\delta_{3}[\rho(x-t)] r(t)-\rho \delta_{2}[\rho(x-t)] p(t)\right\}[A(t, \rho)+z(t, \rho)] e^{\omega_{3} \rho t} d t,
$$

where $A(t, \rho)=-\omega_{3}-\omega_{2} \exp \left[\left(\omega_{2}-\omega_{3}\right) \rho t\right]$. Hence

$z(x, \rho)=e^{\left(\omega_{1}-\omega_{3}\right) \rho x}$

$$
-\left(3 \rho^{2}\right)^{-1} \int_{0}^{x}\left\{\delta_{3}[\rho(x-t)] r(t)-\rho \delta_{2}[\rho(x-t)] p(t)\right\}[A(t, \rho)+z(t, \rho)] e^{-\omega_{0} \rho(x-t)} d t .
$$

Now for fixed $\rho \epsilon S_{1}|z(x, \rho)|$ attains its upper bound $m(\rho)$ on $0 \leq x \leq 1$; hence by (1.3)

$$
\begin{aligned}
m(\rho) \leq 1+ & \left(3|\rho|^{2}\right)^{-1} \int_{0}^{1}\left|\delta_{3}[\rho(x-t)] A(t, \rho) e^{-\omega_{3} \rho(x-t)} r(t)\right| d t \\
& +\left(3|\rho|^{2}\right)^{-1} \int_{0}^{1}\left|\delta_{3}[\rho(x-t)] z(t, \rho) e^{-\omega_{2} \rho(x-t)} r(t)\right| d t \\
& +(3|\rho|)^{-1} \int_{0}^{1}\left|\delta_{2}[\rho(x-t)] A(t, \rho) e^{-\omega_{2} \rho(x-t)} p(t)\right| d t \\
& +(3|\rho|)^{-1} \int_{0}^{1}\left|\delta_{2}[\rho(x-t)] z(t, \rho) e^{-\omega_{3} \rho(x-t)} p(t)\right| d t \\
\leq 1+ & m(\rho)\left(\frac{r}{|\rho|^{2}}+\frac{p}{|\rho|}\right)+2\left(\frac{r}{|\rho|^{2}}+\frac{p}{|\rho|}\right)
\end{aligned}
$$

where

$$
r=\int_{0}^{1}|r(t)| d t, \quad p=\int_{0}^{1}|p(t)| d t
$$

Hence

$$
m(\rho) \leq \frac{1+2\left(r /|\rho|^{2}+p /|\rho|\right)}{1-\left(r /|\rho|^{2}+p /|\rho|\right)}
$$

for $|\rho|$ sufficiently large, from which the lemma follows.

Theorem 1. There exist an infinite number of real characteristic values $\lambda_{n}$ for the system (1); more precisely, there exists a real $\lambda_{k}$ such that all $\lambda_{n}$ with $R\left(\lambda_{n}\right)>\lambda_{k}$ are necessarily real. $^{3}$

Proof. From (1.1) by differentiation,

$$
\begin{aligned}
u^{\prime \prime}(x, \rho)=\rho^{2} \delta_{1}(\rho x) & -\frac{1}{3} \int_{0}^{x}\left\{\delta_{1}[\rho(x-t)] r(t)-\rho \delta_{3}[\rho(x-t)] p(t)\right\} u(t, \rho) d t \\
& -p(x) u(x, \rho),
\end{aligned}
$$

3If $z=x+i y$, then $R(z)=x$ defines the notation $R(z)$ which will be used throughout. 
and the characteristic equation, $u^{\prime \prime}(1, \rho)=0$, becomes

$$
\begin{aligned}
\rho^{2} \delta_{1}(\rho) & -\frac{1}{3} \int_{0}^{1}\left\{\delta_{1}[\rho(1-t)] r(t)-\rho \delta_{3}[\rho(1-t)] p(t)\right\}[A(t, \rho)+z(t, \rho)] e^{\omega_{3} \rho t} d t \\
& -p(1)[A(1, \rho)+z(1, \rho)] e^{\omega_{3} \rho}=0 .
\end{aligned}
$$

Define

$$
\begin{gathered}
E(\rho)=\left(3 \rho^{2}\right)^{-1} \int_{0}^{1}\left\{\delta_{1}\left[\rho(1-t) r(t)-\rho \delta_{3}[\rho(1-t)] p(t)\right\}[A(t, \rho)+z(t, \rho)] e^{-\omega_{\rho} \rho(1-t)} d t\right. \\
+p(1)[A(1, \rho)+z(1, \rho)] \rho^{-2} .
\end{gathered}
$$

It is easily verified that the zeros of $\delta_{1}(\rho)$ are the zeros of $\exp (-3 \rho / 2)+2 \cos \left[3^{1 / 2} \rho / 2\right]$ and are all real for $\rho \epsilon S_{1}$.

It will now be shown that $\left|\rho^{2} \delta_{1}(\rho)\right|>\left|\rho^{2} e^{\omega s \rho} E(\rho)\right|$ for $\rho$ on any one of a set of contours $C_{n}$ in the $\rho$ plane which are trapezoids whose bases are the segments

$$
\begin{array}{ll}
R(\rho)=2(n+1) \frac{\pi}{3^{1 / 2}}, & |\arg \rho| \leq \frac{\pi}{3} ; \\
R(\rho)=2(n+2) \frac{\pi}{3^{1 / 2}}, & |\arg \rho| \leq \frac{\pi}{3} ;
\end{array}
$$

$n=k, k+1, \cdots, k$ sufficiently large. It is sufficient to show this inequality for the upper half of the contour $C_{n}$; namely $C_{n}^{\prime}=C_{n} \cap S_{1}$. This is true because $u(x, \rho)$ is real for real $\rho$ and hence $u\left(x, \rho^{*}\right)=[u(x, \rho)]^{*}$, the star denoting the complex conjugate. Hence $u^{\prime \prime}\left(x, \rho^{*}\right)=\left[u^{\prime \prime}(x, \rho)\right]^{*}$, and consequently $\rho^{2}\left[\delta_{1}(\rho)-e^{\omega{ }^{\circ \rho}} E(\rho)\right]$ takes on values on $C_{n}^{\prime \prime}=C_{n} \cap S_{2}$, the lower half of $C_{n}$, which are the complex conjugates of those taken on on $C_{n}^{\prime}$, the upper half. Set $F(\rho)=\rho^{2} \delta_{1}(\rho), G(\rho)=-\rho^{2} e^{\omega s \rho} E(\rho)$; then since $F\left(\rho^{*}\right)+G\left(\rho^{*}\right)=[F(\rho)]^{*}+[G(\rho)]^{*}$ and $F\left(\rho^{*}\right)=[F(\rho)]^{*}$, we have $G\left(\rho^{*}\right)=[G(\rho)]^{*}$, and hence $\rho^{2} e^{\omega_{3} \rho} E(\rho)$ assumes values on $C_{n}^{\prime \prime}$ which are the complex conjugates of those it takes on on $C_{n}^{\prime}$. It clearly suffices, then, to show that

$$
\left|e^{-\omega s \rho} \delta_{1}(\rho)\right|>|E(\rho)| \quad \text { for } \rho \text { on } C_{n}^{\prime} .
$$

On $\rho=\alpha\left(1+3^{1 / 2} i\right)$ we have

$$
\begin{gathered}
\left|e^{-\omega_{3 \rho} \rho} \delta_{1}(\rho)\right|=\left|2 e^{-3 \alpha} \cos \left(3^{1 / 2} \alpha\right)+1\right| \\
\left|I_{1}\right| \leq \frac{1}{12 \alpha^{2}} 3(2+m) \int_{0}^{1}|r(t)| d t=\frac{1}{4 \alpha^{2}}(2+m) r
\end{gathered}
$$

where

$$
\begin{aligned}
I_{1}=\frac{1}{3 \rho^{2}} \int_{0}^{1}\left\{\exp \left[\left(\omega_{1}-\omega_{3}\right) \rho(1-t)\right]\right. \\
\left.\quad+\exp \left[\left(\omega_{2}-\omega_{3}\right) \rho(1-t)\right]+1\right\}[A(t, \rho)+z(t, \rho)] r(t) d t \\
\quad\left|I_{2}\right| \leq \frac{1}{6 \alpha} 3(2+m) \int_{0}^{1}|p(t)| d t=\frac{1}{2 \alpha}(2+m) p
\end{aligned}
$$


where

$$
\begin{gathered}
I_{2}=\frac{1}{3 \rho} \int_{0}^{1}\left\{\exp \left[\left(\omega_{1}-\omega_{3}\right) \rho(1-t)\right]\right. \\
\left.-\omega_{2} \exp \left[\left(\omega_{2}-\omega_{3}\right) \rho(1-t)\right]-\omega_{3}\right\}[A(t, \rho)+z(t, \rho)] p(t) d t ; \\
\left|I_{3}\right| \leq \frac{|p(1)|}{4 \alpha^{2}}\left(1+e^{-3 \alpha}+m\right),
\end{gathered}
$$

where

$$
I_{3}=p(1)[A(1, \rho)+z(1, \rho)] \rho^{-2} .
$$

On $\rho=2(n+1) \pi / 3^{1 / 2}+i \beta, \beta \geq 0$, we have

$$
\begin{aligned}
\left|e^{-\omega_{s} \rho} \delta_{1}(\rho)\right|=\mid & \exp \left\{-\frac{3}{2}\left[2(n+1) \frac{\pi}{3^{1 / 2}}+i \beta\right]+\frac{3^{1 / 2} i}{2}\left[2(n+1) \frac{\pi}{3}+i \beta\right]\right\} \\
& \quad+\exp \left\{3^{1 / 2} i\left[2(n+1) \frac{\pi}{3^{1 / 2}}+i \beta\right]\right\}+1 \mid \\
= & \mid \exp \left[-3^{1 / 2}(n+1) \pi\right] \exp \left(-\frac{3}{2} i \beta\right)(-1)^{n} \exp \left(-\frac{3^{1 / 2}}{2} \beta\right) \\
& +\exp \left(-3^{1 / 2} \beta\right)+1 \mid
\end{aligned}
$$

hence $\left|e^{-\omega s \rho} \delta_{1}(\rho)\right| \geq 1-\exp \left(3^{1 / 2} \pi\right)$; also

$$
\begin{aligned}
& \left|I_{1}\right| \leq \frac{9}{4} \frac{(2+m) r}{[2(n+1) \pi]^{2}+3 \beta^{2}} \\
& \left|I_{2}\right| \leq \frac{3^{1 / 2}}{2} \frac{(2+m) p}{\left\{[2(n+1) \pi]^{2}+3 \beta^{2}\right\}^{1 / 2}} \\
& \left|I_{3}\right| \leq \frac{3|p(1)|\left(1+\exp \left(-3^{1 / 2} \beta\right)+m\right)}{[2(n+1) \pi]^{2}+3 \beta^{2}} .
\end{aligned}
$$

Now $|E(\rho)| \leq\left|I_{1}\right|+\left|I_{2}\right|+\left|I_{3}\right|$, and clearly for $|\rho|$ large enough there exists $k$ such that for $n=k, k+1, \cdots,(1.4)$ holds. We have then $\left|\rho^{2} \delta_{1}(\rho)\right|>\left|\rho^{2} e^{\omega_{3} \rho} E(\rho)\right|$ on $C_{n}$. Now it is easily verified that there is just one zero of $\delta_{1}(\rho)$ inside $C_{n}$. Hence, by Rouché's theorem, there is just one root of the characteristic equation inside $C_{n}$, and since these roots must occur in complex conjugate pairs, this root is real. This proves the theorem.

By a suitable modification of the choice of contours $C_{n}$ in the proof of the preceding theorem, the following result may be obtained.

Theorem 2. Let the zeros of $\delta_{1}(\rho)$ be denoted by $\rho_{n}^{(0)}$ such that $\rho_{0}^{(0)}<\rho_{1}^{(0)}<\cdots$. Let $\delta>0$ be given, and define $R_{n}$ to be the circle $\left|\rho-\rho_{n}^{(0)}\right| \leq \delta$. Then for $k$ large enough, each root $\rho_{n}$ of the characteristic equation of (1) such that $R\left(\rho_{n}\right)>2 k \pi / 3^{1 / 2}$ lies inside one of the circles $R_{n}, n=k, k+1, \cdots$, there being exactly one $\rho_{n}$, necessarily real, in each such $R_{n}$. 
The proof will be omitted. ${ }^{4}$

From the details of the proof of Theorem 1, it may be seen that for $r, p$, and $|p(1)|$ small enough, $k$ may be chosen equal zero. The question of whether or not in this case all characteristic values of (1) are real, then, reduces to the question of whether or not all roots $\rho_{n}$ of the characteristic equation for which $R\left(\rho_{n}\right)<2 \pi / 3^{1 / 2}$ are real. By means of the following lemma, we obtain a lower bound on $\left|\lambda_{n}\right|$; e.g., $\left|\rho_{n}\right|$; and then by means of the subsequent theorem, apply the arguments of Theorem 1 to a supplementary contour which borders on $C_{0}$ and extends to the region for which no $\rho_{n}$ can occur.

Lemma. Let $\lambda$ be a characteristic value of (1). Then $|\lambda|^{2} \geq 6-\left(m_{1}+m_{2}\right)$ where $|p(t)|^{2} \leq m_{1},|q(t)|^{2} \leq m_{2}$.

Proof. Let $v(x)=u^{\prime}(x)$. Then by (1)

Hence

$$
v^{\prime \prime}(x)=-\left[p(x) u^{\prime}(x)+q(x) u(x)+\lambda u(x)\right], \quad v(0)=v^{\prime}(1)=0 .
$$

$$
v(x)=\sum_{n} \frac{c_{n}+b_{n}+\lambda a_{n}}{\mu_{n}} \phi_{n}(x)
$$

where $\phi_{n}(x)$ and $\mu_{n}$ are the characteristic functions and numbers respectively of the system

and

$$
\phi^{\prime \prime}(x)+\mu \phi(x)=0, \quad \phi(0)=\phi^{\prime}(1)=0
$$

$$
a_{n}=\int_{0}^{1} u(t) \phi_{n}(t) d t, \quad b_{n}=\int_{0}^{1} u(t) q(t) \phi_{n}(t) d t, \quad c_{n}=\int_{0}^{1} u^{\prime}(t) p(t) \phi_{n}(t) d t .
$$

Hence by (1.5) and integration from 0 to $x$, one obtains

$$
u(x)=\sum_{n} \frac{c_{n}+b_{n}+\lambda a_{n}}{\mu_{n}} z_{n}(x) \quad \text { where } \quad z_{n}(x)=\int_{0}^{x} \phi_{n}(t) d t .
$$

Multiplying by $\phi_{k}(x)$ and integrating from 0 to 1 one obtains

$$
a_{k}=\int_{0}^{1} \phi_{k}(x) u(x) d x=\sum_{n} \frac{c_{n}+b_{n}+\lambda a_{n}}{\mu_{n}} \int_{0}^{1} z_{n}(x) \phi_{k}(x) d x .
$$

Hence, using Schwarz's inequality,

where

$$
\left|a_{k}\right|^{2} \leq M \sum_{n}\left(\left|c_{n}\right|^{2}+\left|b_{n}\right|^{2}+|\lambda|^{2}\left|a_{n}\right|^{2}\right)\left|\int_{0}^{1} z_{n}(x) \phi_{k}(x) d x\right|^{2}
$$

$$
M=\sum_{n} \frac{1}{\mu_{n}^{2}}=\frac{1}{6} .
$$

Summing over $k$ and using Parseval's theorem:

$$
\begin{aligned}
\sum_{k}\left|a_{k}\right|^{2} & \leq M \sum_{n}\left(\left|c_{n}\right|^{2}+\left|b_{n}\right|^{2}+|\lambda|^{2}\left|a_{n}\right|^{2}\right) \sum_{k}\left|\int_{0}^{1} z_{n}(x) \phi_{k}(x) d x\right|^{2} \\
& =M \sum_{n}\left(\left|c_{n}\right|^{2}+\left|b_{n}\right|^{2}+|\lambda|^{2}\left|a_{n}\right|^{2}\right) \int_{0}^{1}\left|z_{n}(x)\right|^{2} d x .
\end{aligned}
$$

${ }^{4}$ See L. E. Ward, [4] pp. 419-420. 
However, since

$$
\int_{0}^{1}\left|z_{n}(x)\right|^{2} d x \leq \int_{0}^{1}\left\{\int_{0}^{1}\left|\phi_{n}(t)\right|^{2} d t\right\} d x=1,
$$

and since we may assume

$$
\int_{0}^{1}|u(x)|^{2} d x=1
$$

another application of Parseval's theorem to (1.6) gives

$$
1 \leq M\left\{\int_{0}^{1}\left|p(t) u^{\prime}(t)\right|^{2} d t+\int_{0}^{1}|q(t) u(t)|^{2} d t+|\lambda|^{2}\right\} .
$$

Let $|p(t)|^{2} \leq m_{1},|q(t)|^{2} \leq m_{2}$; then from (1.7) one has

$$
1 \leq M\left\{m_{1} \int_{0}^{1}\left|u^{\prime}(t)\right|^{2} d t+m_{2}+|\lambda|^{2}\right\}
$$

Multiplying (1.5) by $\phi_{k}(x)$ and integrating from 0 to 1 , one obtains

$$
\left|\int_{0}^{1} \phi_{k}(x) u^{\prime}(x) d x\right|^{2}=\left|\frac{c_{k}+b_{k}+\lambda a_{k}}{\mu_{k}}\right|^{2} \leq \frac{\left|c_{k}\right|^{2}+\left|b_{k}\right|^{2}+|\lambda|^{2}\left|a_{k}\right|^{2}}{\mu_{k}^{2}}
$$

and summing over $k$ and applying Parseval's theorem again:

$$
\int_{0}^{1}\left|u^{\prime}(x)\right|^{2} d x \leq M\left(m_{1} \int_{0}^{1}\left|u^{\prime}(x)\right|^{2} d x+m_{2}+|\lambda|^{2}\right)
$$

from which it follows that

$$
\left(1-M m_{1}\right) \int_{0}^{1}\left|u^{\prime}(x)\right|^{2} d x \leq M\left(m_{2}+|\lambda|^{2}\right) .
$$

Hence, by (1.8) and (1.9):

$$
\left(1-M m_{1}\right) \leq M\left[m_{1} M\left(m_{2}+|\lambda|^{2}\right)+\left(m_{2}+|\lambda|^{2}\right)\left(1-M m_{1}\right)\right]=M\left(m_{2}+|\lambda|^{2}\right) \text {. }
$$

From this one obtains

$$
\frac{1-M\left(m_{1}+m_{2}\right)}{M} \leq|\lambda|^{2} .
$$

Since $M=1 / 6$, the proof of the lemma is complete.

Theorem 3. Let p, $r, m_{1}, m_{2}$ be defined as in previous theorems and lemmas. Then if these constants and $|p(1)|$ are sufficiently small, all characteristic values of the system (1) are real.

Proof. It has already been noted that for sufficiently small values of $p, r$, and $|p(1)|$, $k$ may be chosen equal zero. We now consider the supplementary contour $C_{-1}$, a trapezoid whose bases are the segments:

$$
\begin{array}{ll}
R(\rho)=\frac{2 \pi}{3^{1 / 2}}, & |\arg \rho| \leq \frac{\pi}{3} ; \\
R(\rho)=\frac{R}{2}, & |\arg \rho| \leq \frac{\pi}{3} ;
\end{array}
$$


where $R=\left[6-\left(m_{1}+m_{2}\right)\right]^{1 / 6}$. By Theorem 1 and the lemma all non-real characteristic values $\lambda_{n}=\rho_{n}^{3}$ must be such that $\rho_{n}$ is in or on $C_{-1}$. However, for sufficiently small $p$, $r, m_{1}, m_{2}$, and $|p(1)|$, the argument used to prove Theorem 1 is applicable to $C_{-1}$ and we conclude that there is just one root $\rho_{-1}$, necessarily real, inside $C_{-1}$. This proves the theorem.

\section{PART II}

Consider the system

$$
\left[f(t) y^{\prime \prime}(t)\right]^{\prime}+\lambda g(t) y(t)=0, \quad y(0)=y^{\prime}(0)=y^{\prime \prime}(1)=0,
$$

$f$ and $g$ real and analytic on $0 \leq t \leq 1, f(t)>0, g(t)>0$. The transformation

$$
x=\frac{1}{d} \int_{0}^{t} h(s) d s \quad \text { where } \quad h(s)=\left[\frac{f(s)}{g(s)}\right]^{1 / 3}, \quad d=\int_{0}^{1} h(s) d s,
$$

has an inverse $t=t(x)$. This inverse transforms (2) into

$$
\begin{gathered}
\frac{d^{3}}{d x^{3}} y[t(x)]+p_{1}(x) \frac{d^{2}}{d x^{2}} y[t(x)]+p_{2}(x) \frac{d}{d x} y[t(x)]+y[t(x)]=0, \\
y(0)=y^{\prime}(0)=y^{\prime \prime}(1)=0
\end{gathered}
$$

where

$$
\begin{aligned}
& p_{1}(x)=\frac{1}{d}\left\{3 h^{4}[t(x)] h^{\prime}[t(x)]+\frac{f^{\prime}[t(x)]}{g[t(x)]} h^{2}[t(x)]\right\} \\
& p_{2}(x)=\frac{1}{d}\left\{h^{3}[t(x)] h^{\prime \prime}[t(x)]+\frac{f^{\prime}[t(x)]}{f[t(x)]} h^{\prime}[t(x)]\right\},
\end{aligned}
$$

the notation $f^{\prime}[t(x)]$ meaning that the substitution $t=t(x)$ has been made in the function of $t: f^{\prime}(t)$.

Next, the substitution

$$
y[t(x)]=u(x) \exp \left[-\frac{1}{3} \int_{0}^{x} p_{1}(s) d s\right]
$$

and subsequent multiplication by

$$
\exp \left[\frac{1}{3} \int_{0}^{x} p_{1}(s) d s\right]
$$

reduces $(2.1)$ to $u^{\prime \prime \prime}(x)+p(x) u^{\prime}(x)+[q(x)+\lambda] u(x)=0, u(0)=u^{\prime}(0)=u^{\prime \prime}(1)=0$, where

$$
\begin{aligned}
& p(x)=p_{2}(x)-\frac{p_{1}^{2}(x)}{3}-p_{1}^{\prime}(x), \\
& q(x)=\frac{2}{27} p_{1}^{3}(x)-\frac{p_{1}^{\prime \prime}(x)}{3}-\frac{p_{1}(x) p_{2}(x)}{3} .
\end{aligned}
$$

Hence the results of Part I apply to the system (2). 
Noting the structure of $p$ and $q$; e.g., $p_{1}$ and $p_{2}$, in terms of $f$ and $g$, we state an obvious corollary of Theorem 3.

Corollary. Let $\left|f^{(n)}(t)\right|<\epsilon,\left|g^{(n)}(t)\right|<\epsilon$ for $n=1,2$, 3, 4. Then for $\epsilon$ sufficiently small, all the characteristic values of (2) are real.

It is also clear from the structure of $p$ and $q$ that less restrictive, although perhaps more complicated, conditions on $f$ and $g$ than those in the hypothesis of the above corollary will yield the same conclusion.

The author wishes to thank Professors W. Feller and W. R. Sears for suggesting the problem and for helpful suggestions toward its solution.

\section{REFERENCES}

[1] A. H. Flax, Aeroelastic problems at supersonic speed, Proc. 2nd International Aeronautics Conference, I.Ae.S., New York, pp. 322-360.

[2] H. K. Cheng, A variational method for the differential equation arising from aeroelastic problems, Thesis, Graduate School of Aeronautical Engineering, Cornell University, Feb. 1950.

[3] L. E. Ward, Some third order irregular boundary value problems, Trans. Am. Math. Soc. 29, 716-745 (1927).

[4] L. E. Ward, A third order irregular boundary value problem and the associated series, Trans. Am. Math. Soc. 34, 417-434 (1932).

\section{ON AN EQUATION OCCURRING IN THE HARMONIC ANALYSIS OF VISCOUS FLUID FLOW*}

\section{BY RICHARD BELLMAN (Stanford University)}

1. Introduction. It was shown by J. Kampé de Feriet ${ }^{1}$ that the Fourier transform

$$
z\left(w_{1}, w_{2}, t\right)=\frac{1}{4 \pi^{2}} \int_{-\infty}^{\infty} \int \zeta(x, y) \exp \left[-i\left(w_{1} x+w_{2} y\right)\right] d x d y
$$

of the vorticity, $\zeta(x, y)$, associated with the two-dimensional flow of an incompressible fluid extending over the entire $(x, y)$-plane, under mild conditions, satisfies the nonlinear integro-differential equation

$$
\begin{aligned}
& \frac{\partial}{\partial t} z\left(w_{1}, w_{2}, t\right)=-v\left(w_{1}^{2}+w_{2}^{2}\right) z\left(w_{1}, w_{2}, t\right) \\
& \quad+2 \int_{-\infty}^{\infty} \int\left(\frac{\theta_{1} w_{2}-\theta_{2} w_{1}}{\theta_{1}^{2}+\theta_{2}^{2}}\right) z\left(\theta_{1}, \theta_{2}, t\right) \bar{z}\left(\theta_{1}+w_{1}, \theta_{2}+w_{2}, t\right) d \theta_{1} d \theta_{2},
\end{aligned}
$$

and the boundary condition

$$
z\left(w_{1}, w_{2}, 0\right)=\phi\left(w_{1}, w_{2}\right)
$$

${ }^{*}$ Received November 8,1950 . The results contained in this paper were obtained in connection with research sponsored by the Rand Corporation.

${ }^{1} \mathrm{~J}$. Kampé de Feriet, Harmonic analysis of the two-dimensional flow of an incompressible viscous fluid, Q. Appl. Math. 6, 1-13 (1948). 